\begin{tabular}{|c|l|}
\hline Title & Phosphorescence decay time measurements using intensity correlation spectroscopy. \\
\hline Author(s) & Nishimura, Goro; Pack, Chan-Gi; Tamura, Mamoru \\
\hline Citation & $\begin{array}{l}\text { Experimental and Molecular Pathology, 82(2), 175-183 } \\
\text { https://doi.org/10.1016/.y exmp.2007.01.004 }\end{array}$ \\
\hline Issue Date & 2007-04 \\
\hline Doc URL & http://hdl.handle.net/2115/26222 \\
\hline Type & article (author version) \\
\hline File Information & EMP82-2.pdf \\
\hline
\end{tabular}

Instructions for use 
Invited Article for the Half Special Issue of Experimental and Molecular Pathology

"Exploring the Biomedical Applications of Microscopy and Spectroscopy"

Guest Editor Z.Földes-Papp

\title{
Phosphorescence decay time measurements using intensity
}

\section{correlation spectroscopy}

\author{
Goro Nishimura $^{1}$, Chan-Gi Pack ${ }^{1,2}$ and Mamoru Tamura ${ }^{1}$ \\ ${ }^{1)}$ Biophysics Laboratory, Research Institute for Electronic Science, \\ Hokkaido University, N12W6, Sapporo 060-0812, Japan \\ ${ }^{2}$ The 21st COE program, "Topological Science and Technology", \\ Hokkaido University, N13W8, Sapporo 060-8628, Japan.
}

\section{Corresponding author:}

Goro Nishimura

Biophysics Laboratory, Research Institute for Electronic Science, Hokkaido University, N12W6, Sapporo 060-0812, Japan

Tel:+81-11-706-3371 Fax:+81-11-706-4964 E-mail: gnishi@imd.es.hokudai.ac.jp 


\begin{abstract}
In this paper, we report on a phosphorescence measurements for oxygen dynamics in cells by means of a correlation method, which is an expansion of the fluorescence correlation spectroscopy. The intensity correlation function of the emission excited by a pulsed light source was measured. With changing the pulse timing, both the fluorescence correlation function and the decay time of phosphorescence could be analyzed. This method was applied for the analysis of the oxygen dynamics in HeLa cells stained by Pd(II)-porphine. The decay function consisted of two exponential components, which might be attributed to free and protein-bound forms of Pd(II)-porphine in the cell, respectively. The relative change of the oxygen concentration under normal and uncoupled respiration conditions was also measured. The simplicity of this method is a great advantage in the biological applications. Although the current system we used was limited in the temporal resolution, the method is in principal applicable to faster decay time measurements down to the nano-second range of the fluorescence decay times.
\end{abstract}

\title{
Keywords
}

fluorescence correlation spectroscopy, phosphorescence, lifetime, oxygen dynamics, Pd porphine, protoporphyrin IX, cell imaging 


\section{Introduction}

The fluorescence correlation spectroscopy (FCS) is one of the most popular application for monitoring the molecular interactions at the single molecule level and now widely used in biological research. The most popular FCS method is based on the analysis of fluorescence intensity fluctuation due to the random motion of fluorescent particles in a small region of a sample.(Rigler and Elson, 2001) FCS is a very high sensitive and noninvasive method to detect a very small region defined by the confocal optics, and applicable to the analysis of dynamic behaviors and interactions not only in in vitro systems but also in living cells.(Bacia et al., 2006; Vukojevi et al., 2005) Moreover, although FCS method is basically a statistical ensemble one, true single molecule in solution can also be analyzed.(Földes-Papp, 2006)

Many researchers are now interested in FCS applications for much more complex targets, such as in tissue, organ and in vivo systems, and applications from basic science to clinical purpose. For this, a combination with other measurement modalities, for example, such as the luminescence decay measurements, is very useful. The decay profile is very sensitive to the environment near the chromophore, to which FCS measurements are relatively insensitive. The decay measurement can be a very powerful tool to understand the molecular interactions.(Lakowicz, 1999) The combination with the decay time measurement system is recently developed.(Felekyan et al., 2005) Usually, the decay time measurements require different detection system, such as the time-correlated single photon counting (TCSPC) system and the pulsed or modulated light source. Therefore, the extension of the commercially available FCS system tends to become very complex and expensive.

Here we focus on phosphorescence decay measurements at the cellular level using a conventional FCS system as a pilot study. The phosphorescence are well studied and there are many 
applications to devices,(Chou and Chi, 2007) protein chemistry(Banks and Kerwin, 2004; Bódis et al., 2004; Pravinata et al., 2005) and physiology.(Mik et al., 2004) The phosphorescence decay time is very sensitive to the environment of phosphorescence probes. In particular, since the phosphorescence is strongly quenched by molecular oxygen, the oxygen concentration in tissue from cellular level can be determined by the decay measurements. The oxygen dynamics in tissue has been studied in numerous works and the phosphorescence measurement is a very popular method.(Wilson and Vinogradov, 2003)

In such works, some basic issues in oxygen dynamics at cellular level, such as the oxygen concentration barrier or gradient across the membrane, have not been well understood. Furthermore, some interesting biological responses - for example, the signal transaction and the apoptoic response under hypoxic condition - have not well anlyzed. These issues may be analyzed using both oxygen concentration monitoring and interaction analysis by FCS in the cellular level.

Recently, we have found that HeLa cells could be stained by Pd(II)-porphine and its phosphorescence decay in HeLa cells could be measured by a correlation measurement.(Nishimura et al., 2007) On the other hand, the new possibility using the delayed fluorescence of endogenous protoporphyrin IX (PpIX) for the oxygen monitoring in cells has been demonstrated.(Mik et al., 2006) These indicate a possibility of the decay analysis for the oxygen dynamics measurement in the cellular level.

In this paper, we demonstrate a decay measurement with the same detection and analysis system using a conventional FCS system but with a pulsed excitation source instead of a continuous wave laser. We have briefly reported our system.(Nishimura et al., 2007) Here, we demonstrate a correlation method for the phosphorescence decay time measurements using a HeLa cell system. 
We focus on both phosphorescence decay and fluorescence correlation measurements at single cell level using a single setup. We conduct oxygen concentration measurements in HeLa cells. We concentrate on the methodological point of the correlation method instead of the biological optimizations in the measurements. Finally, we discuss the future applications in biology and medicine.

\section{Materials and Methods}

We prepared a $6.5 \mathrm{mM}$ stock solution of 5,10,15,20-tetrakis(4-sulfonatophenyl)-porphine (Pd:TSPP; Porphyrin Systems GbR, Luebeck, Germany) in phosphate-buffered saline (PBS) and stocked at room temperature. Finally, the Pd:TSPP solution was diluted to about $20 \mu \mathrm{M}$ by PBS or dissolved to $2.5 \%$ bovine serum albumin (BSA) in PBS for the solution measurements. The solution about $1.5 \mathrm{ml}$ in a small plastic chamber covered with a cover slip of microscope for sealing. All measurements were conducted under room temperature at $24-27^{\circ} \mathrm{C}$.

The oxygen in the solution was removed by a glucose-oxidase-catalase (GOx-Cat) system in both solution and cell measurements.(Vanderkooi et al., 1987) The medium was consisted of 50 to $200 \mathrm{nM}$ glucose oxidase (GOx, EC1.1.3.4, G6641, Sigma), $20 \mathrm{mM}$ D-glucose, and $0.1 \mu \mathrm{M}$ catalase (Cat, EC1.11.1.6, C9322, Sigma).

In the cell measurements, HeLa cells were incubated until confluent in a $3.5 \mathrm{~cm}$ glass based dish (Iwaki 3911-035, Asahi Techno Glass, Chiba, Japan), which had a small dip (12 mm in diameter, about $140 \mu \mathrm{l}$ in volume) for microscope measurements. Before the experiment, the cells were incubated with a $1 \mathrm{mM}$ Pd:TSPP solution for about $1 \mathrm{hr}$, rinsed with PBS, and then the medium was replaced by OptiMEM buffer at $37^{\circ} \mathrm{C}$. The phosphorescence images were obtained 
by a laser scanning confocal microscope (LSM510, Zeiss, Jena, Germany) with an excitation at $514.5 \mathrm{~nm}$ and $530 \mathrm{~nm}$ LP filer. In the phosphorescence measurements, the dip in the dish was covered with a microscope cover slip to seal the sample, and then the dish was put upside down on the sample stage for phosphorescence decay measurements.

The instrumentation has been reported.(Nishimura et al., 2007) Briefly, an epifluorescence microscope (BX-50, Olympus, Tokyo, Japan) was modified for pulsed excitation using a frequency doubled Q-switched $\mathrm{Nd}^{3+}$ :YAG laser (LCS-DTL-314QT, Laser Compact Co.Ltd., Moscow, Russia). The laser pulses were synchronized to a random or periodical trigger source. The random trigger was used in phosphorescence measurements. On the other hand, the periodical trigger $(50 \mathrm{KHz})$ was only used in fluorescence correlation measurements. The random trigger was generated by dark count pulses from a photomultiplier tube so that the trigger timing was randomized. The laser light was focused into the sample using an objective lens (UPlanApo x60, NA 1.2/water, or UPlanFl x10, NA 0.3, Olympus). A standard green fluorescence filter set (U-MWG2, Olympus) was used for the phosphorescence detection. A $62.5 \mu \mathrm{m}$ graded index fiber (NA 0.27) was positioned at the focus plane of a camera port of the microscope and delivered the emission to an avalanche single photon counting module (SPCM-100, RCA). The arrival times of photon were recorded and the intensity correlation function was calculated after measurements.

In the phosphorescence measurements in vitro, the lower magnification lens was used mainly. On the other hand, the higher one was used in the cell measurements and fluorescence correlation measurements. The excitation position in a cell was visually guided by eyes seeing both the excitation laser spot and the transilluminated image simultaneously. The organella, such as nucleus and cytoplasm, could be arbitrarily selected. 


\section{Analysis}

The correlation function of the emission intensity $I(t)$ is calculated by

$$
g(\tau)=\frac{\langle I(t) I(t+\tau)\rangle}{\langle I(t)\rangle^{2}}-1
$$

where $\langle\cdots\rangle$ denotes an ensemble average. For the single exponential decay of the emission, the correlation function can be obtained as

$$
g(\tau)=\frac{1}{2 v \tau_{0}} \exp \left(-\frac{\tau}{\tau_{0}}\right)
$$

where $v$ is the average excitation rate, when the timing of the excitation is randomized. Furthermore, the correlation function of double exponential decay system, $I(t)=a_{0} / \tau_{0} \exp \left(-t / \tau_{0}\right)+$ $a_{1} / \tau_{1} \exp \left(-t / \tau_{1}\right)$, where $a_{0}+a_{1}=1$, is expressed by

$$
g(\tau)=\frac{1}{2 v}\left\{\left[1+\frac{a_{1}(1-\gamma)}{1+\gamma}\right] \frac{a_{0}}{\tau_{0}} \exp \left(-\frac{\tau}{\tau_{0}}\right)+\left[1-\frac{a_{0}(1-\gamma)}{1+\gamma}\right] \frac{a_{1}}{\tau_{1}} \exp \left(-\frac{\tau}{\tau_{1}}\right)\right\}
$$

where $\gamma=\tau_{1} / \tau_{0}$. The detailed derivation of the correlation function is given in Appendix.

From Eq.(3), the decay time of each decay component is identical to the decay time in the fluorescence decay function. On the other hand, the fraction of the exponential term with the longer decay time in the correlation function is always larger than the fraction in the decay kinetic function. Namely, the fraction in the correlation function is overestimated for the fraction of the slower decay term.

A two-component correlation function, which was mainly used in our analysis in cell measurements, can be expressed as

$$
g(\tau)=\alpha\left[f \exp \left(-\tau / \tau_{0}\right)+(1-f) \exp \left(-\tau / \tau_{1}\right)\right]
$$


where $\alpha$ and $f$ are the normalization constant and the fraction on an interval of $[0: 1]$, respectively. The pre-exponential factor of the decay kinetics function $a_{0}$ is related to $f$ as

$$
a_{0}=\frac{1-\left[1-(1-\gamma)(1+\gamma) f^{\prime}\right]^{1 / 2}}{1-\gamma}
$$

where $f^{\prime}=f /[(1-f) \gamma+f]$, or

$$
f=\frac{2 \gamma a_{0}-(1-\gamma) \gamma a_{0}^{2}}{1+\gamma-2(1-\gamma) a_{0}+(1-\gamma)^{2} a_{0}^{2}} .
$$

In cell measurements, the correlation function consists of two exponential terms. We consider a two-component Stern-Volmer quenching system for the analysis of oxygen dynamics. The first and second decay times are given by $1 / \tau_{i}=1 / \tau_{i}^{0}+k_{i}\left[\mathrm{O}_{2}\right]$, where $k_{i}$ is the Stern-Volmer quenching constant. Here the superscript 0 refers to the parameter without oxygen. Then, the ratio of the decay time can be expressed as,

$$
\gamma=\frac{1+k_{0} \tau_{0}^{0}\left[\mathrm{O}_{2}\right]}{1+k_{1} \tau_{1}^{0}\left[\mathrm{O}_{2}\right]} \gamma_{0},
$$

where $\gamma_{0}=\tau_{1}^{0} / \tau_{0}^{0}$. On the other hand, the amplitude can be written as,

$$
a_{0}=\left[1+\left(\frac{1+k_{0} \tau_{0}^{0}\left[\mathrm{O}_{2}\right]}{1+k_{1} \tau_{1}^{0}\left[\mathrm{O}_{2}\right]}\right)\left(\frac{a_{1}^{0}}{a_{0}^{0}}\right)\right]^{-1} .
$$

In principle, the two-component fitting to the correlation function using Eq.(4) can determine $f$, $\tau_{0}$ and $\tau_{1}$. Eventually, the quenching constants or the oxygen concentration can be determined. However, the fitting is erroneous if the statistics is not good enough and the correlation function is noisy. In our oxygen dynamics measurements, the data statistics was not good under aerobic condition.

When $\tau_{0}^{0}, \tau_{1}^{0}, a_{0}^{0}$ and $a_{1}^{0}$ are known, we can reduce the number of free parameters using SternVolumer relationship, Eqs.(6), (7) and (8). Eventually, three free parameters $f, \tau_{0}$ and $\tau_{1}$ in Eq.(4) 
are reduced to two free parameters $k_{0}\left[\mathrm{O}_{2}\right]$ and $k_{1}\left[\mathrm{O}_{2}\right]$, which can be determined by the fitting. Once the quenching constants are known, the oxygen concentration can be determined.

\section{Results}

\section{Decay time measurements in vitro}

Typical correlation functions of the phosphorescence of a $22 \mu \mathrm{M}$ Pd:TSPP-BSA conjugate in PBS under decreasing oxygen concentration by the GOx-Cat system is shown by Fig.1A. The correlation functions from top to bottom are corresponding to the higher oxygen concentration to lower concentration, respectively. The correlation amplitudes were not corrected by the background count in this figure. The correlation functions below $1 \mu$ s were strongly distorted due to the dead time of the instrument. The correlation curves under higher oxygen concentration were more noisy because of lower count rate. The correlation functions of a $22 \mu \mathrm{M}$ Pd:TSPP solution are also shown in Fig.1B. The correlation functions decay faster than those of the Pd:TSPP-BSA conjugates.

The fitting results by the single exponential decay function in the region over $0.9-1 \mu$ s to $100 \mathrm{~ms}$ are shown by the lines in Figs.1A and 1B. The correlation functions were mostly well fitted. The phosphorescence measurements with the higher magnification lens (x60) were also conducted (data not shown). The decays of the correlation functions did not change. This suggests that the diffusion of Pd:TSPP-BSA and Pd:TSPP in the focusing volume does not affect our decay time measurement.

The correlation amplitude, after the back ground correction using $g_{\text {obs }}(0)\left(I /\left(I-I_{\mathrm{b}}\right)\right)^{2}$ where 
$I$ and $I_{\mathrm{b}}$ are the observed intensity and the background count, respectively, was plotted against the decay time as shown in Fig.1C. The open and closed symbols denote the results by Pd:TSPP and Pd:TSPP-BSA, respectively. Both amplitudes are expressed by a line, $g(0)=a / \tau$. The proportional constant $a=(2 v)^{-1}$ is given by Eq.(2) and the fitting determines $v=55.2 \pm 0.7 \mathrm{sec}^{-1}$. $v$ is fairly in good agreement with the average trigger rate $51.6 \pm 7.2 \mathrm{sec}^{-1}$. The observed correlation function is in good agreement with the theory, and thus the correlation method can be used for the analysis of decay profile. This could be also supported by the simultaneous decay measurement using the GOx-Cat system with an oxygen electrode.(Nishimura et al., 2007)

The decay times of Pd:TSPP-BSA were determined as $0.025 \pm 0.002$ and $0.591 \pm 0.016 \mathrm{~ms}$ under aerobic and anaerobic conditions, respectively. The quenching constant was also determined by the Stern-Volmer relationship as $0.233 \pm 0.006 \mathrm{~ms}^{-1} \mathrm{Torr}^{-1}$. The phosphorescence decay times of Pd:TSPP in PBS were also determined as $0.0025 \pm 0.0004$ and $0.153 \pm 0.004$ ms under aerobic and anaerobic conditions, respectively. The phosphorescence decay time and the quenching constant of Pd:TSPP-BSA conjugate were about 4-times longer and about 1/10 times smaller of Pd:TSPP, respectively. This result is consistent with the previous results using a BSA conjugate Pd-porphine.(Vanderkooi et al., 1987)

\section{FCS capability of the system}

The capability of the fluorescence correlation measurements with our system was demonstrated with fluorescent beads (14 $\mathrm{nm}$ in diameter). In the measurements, the interval of the random excitation was too sparse to measure the diffusion time of the beads. Thus, the highest repetitive frequency of the laser $(50 \mathrm{kHz})$ was used in the measurements. The typical correlation curves are 
shown in Fig.2A. The correlation function with wider time range is also shown in the inset figure and the peaks at a $20 \mu$ s cycle indicates the repetition period of the excitation. The amplitude of these peaks becomes small with larger $\tau$ because we used wider bins at larger $\tau$ in the calculation of the correlation function. The peaks were almost disappeared and the curve became smooth in a slower region over $0.1 \mathrm{~ms}$. The data was analyzed in this smooth region.

The curve was well fitted by a double component 3D free diffusion model, $g(\tau)=1 / n[f(1+$ $\left.\left.\tau / \tau_{1}\right)^{-1}\left(1+\tau / \tau_{1} / q^{2}\right)^{-1 / 2}+(1-f)\left(1+\tau / \tau_{2}\right)^{-1}\left(1+\tau / \tau_{2} / q^{2}\right)^{-1 / 2}\right]$, indicated by the solid line in the figure $\left(n=22.4 \pm 0.3, f=0.62 \pm 0.02, \tau_{1}=3.5 \pm 0.4 \mathrm{~ms}\right.$, and $\tau_{2}=68 \pm 7 \mathrm{~ms} . q=10$ was fixed in the fitting). The first component is considered to be the diffusion of the mono-disperse beads, but the second component might be due to aggregation of beads. The radius $\omega_{0}$, where the detected emission intensity becomes $e^{-2}$ of the peak intensity, could be determined as $0.66 \mu \mathrm{m}$ using $\omega_{0}^{2}=4 D \tau_{1}$ and $D=3.1 \times 10^{-7} \mathrm{~cm}^{2} / \mathrm{s}$.

The correlation amplitude is proportional to the inverse of number of particles in the observation volume. The number of particles estimated from the correlation function as a function of the concentration is shown in Fig.2B. The background was taken into the account of the analysis. As expected, the proportionality of the number of particles to the concentration of beads is clearly confirmed. Thus, the system has a capability for the fluorescence correlation measurements of particles like beads in solution.

\section{Phosphorescence image and correlation function in HeLa cells}

Since above results indicate that the phosphorescence correlation measurement can be utilized to obtain phosphorescence decay times, we have applied this method to the oxygen concentration 
measurements in cells. Before the measurements, we confirmed that HeLa cells could be stained by Pd:TSPP solution using a commercial LSM system as shown in Fig.3A. Interestingly, the nucleus, in particular the nucleolus, were stained strongly. Some of the cells were not or very weakly stained as shown in the inset of Fig.3A. The question remained to be answered about the variety of staining level of the cells, which might be controlled by unknown transport mechanism of Pd:TSPP. The laser excitation in the cells with our system is shown by a digital camera picture at the eye piece of the microscope as shown in Fig.3B. From the transillumination image of the cells, the arbitrary locations, cytoplasm and nucleus, in an individual cell could be selected visually. A laser reflection spot, marked by the arrow in the image, indicates an excitation point in the cytoplasm of a cell, for example.

The correlation functions of the phosphorescence at a point of cytoplasm in a cell under aerobic and anaerobic conditions are shown in Fig.3C. The anaerobic condition was maintained by the GOx-Cat system. On the other hand, the aerobic condition was equilibrated with air using an open chamber. The correlation amplitude is normalized to 1 and the correlation offset is subtracted. Under both conditions, the decays were slower than in the Pd:TSPP solution. Under aerobic condition, the morphology of the cell was sometimes not maintained until the completion of the measurement $(600 \mathrm{sec})$, though the statistics still remained poor. The results by double exponential fittings are shown by solid lines and the fittings are very well in both curves.

\section{Decay time components in HeLa cells}

The decay times at cytoplasm and nucleus under aerobic and anaerobic conditions were analyzed by double exponential fitting and are summarized in Figs.4A-D. Two decay components are well 
separated under anaerobic condition as shown in Figs.4C and 4D. In contrast, the separation is very poor under aerobic condition as shown in Figs. 4A and B. In fact, the correlation function under aerobic condition can be fitted by a single exponential function with almost the same goodness of fit. This might be due to the poor statistics of the aerobic data and lack of data in the dead time distorted time region less than $1 \mu \mathrm{s}$.

Under the aerobic condition, the decay times and the fractions become $0.055 \pm 0.028(46 \pm 18 \%) \mathrm{ms}$ and $0.012 \pm 0.008(54 \pm 18 \%) \mathrm{ms}$ in cytoplasm, and $0.052 \pm 0.024(45 \pm 17 \%)$ and $0.012 \pm 0.009(55 \pm 17 \%) \mathrm{ms}$ in nucleus, respectively. Both analyses clearly indicate no significant difference of the phosphorescence decay between in the cytoplasm and in the nucleus. Under anaerobic condition, the decay times and the fractions are $0.51 \pm 0.07(72 \pm 6 \%)$ and $0.085 \pm 0.040(28 \pm 6 \%) \mathrm{ms}$ in cytoplasm as well as $0.52 \pm 0.05(71 \pm 3 \%)$ and $0.090 \pm 0.034(29 \pm 3 \%)$ in nucleus, respectively. Again, there is no significant difference between the cytoplasm and nucleus.

The slower decay times under anaerobic condition are very similar to the value in BSA conjugated Pd:TSPP solution, suggesting that Pd:TSPP in cells is supposed to be a protein-bound form. The faster component, on the other hand, is similar to the value in solution, suggesting that Pd:TSPP is not bound to proteins or forms a very weak complex with some components. The faster decay time component under aerobic condition is close to the value in free solution, but slightly slower than the value measured in buffer solution. The possibility of lower oxygen concentration inside the cell relative to the medium outside the cell can be excluded because the addition of sodium cyanide solution, which inhibits respiration, did not alter the decay curves within our experimental errors. This result suggests that Pd:TSPP with the faster decay time might weakly interact or be bound to some components inside the cell. 
Using the average decay times and the oxygen partial pressures 150 Torr and 0 Torr under aerobic and anaerobic conditions, respectively, the Stern-Volmer quenching constants can be estimated as $0.11 \mathrm{~ms}^{-1} \mathrm{Torr}^{-1}$ and $0.48 \mathrm{~ms}^{-1} \mathrm{Torr}^{-1}$ for the slow and fast decay time components, respectively. The quenching constant of the slower component is smaller than the value of the faster component. This qualitatively agrees with the measurements of Pd:TSPP and Pd:TSPPBSA conjugates in solution. Note that the uncertainties of the decay time under aerobic condition cause the large errors in estimation of the quenching constants.

\section{Oxygen dynamics measurements in HeLa cells}

The oxygen dynamics in a single cell can be traced by our decay measurement. In the analysis, the record of the photon arrival times during the measurement period $(600-900 \mathrm{sec})$ was divided into some small records (100-200 sec), and then the correlation function was calculated for each small record. The series measurements were interrupted by the data transfer and processing periods. The correlation functions measured in cytoplasm of a single cell with the GOx-Cat system are shown in the inset of the Fig.5A. Assuming the Stern-Volumer relationship, the quenching constants in the cell can be determined by the decay times under two different conditions. From double exponential fitting, decay times $0.58 \mathrm{~ms}(71 \%)$ and $0.093 \mathrm{~ms}(29 \%)$ were obtained when the oxygen was completely consumed. The fraction of the slow component $f=0.71$ in the correlation function yields the fraction of the decay function $a_{0}=0.84$ using Eq.(5).

Using these values, the quenching constants in this cell can be estimated as $0.18 \mathrm{~ms}^{-1} \mathrm{Torr}^{-1}$ and $0.55 \mathrm{~ms}^{-1} \mathrm{Torr}^{-1}$ by the fitting of the correlation function, described in the theory section, at $175 \mathrm{~s}$ after the GOx-Cat mixing. Here we assume a $120 \mathrm{Torr}^{\mathrm{PO}} \mathrm{P}_{2}$. Then, the $\mathrm{PO}_{2}$ in the cell can be 
obtained by fitting with the oxygen concentration as a fitting parameter and with fixing the other parameters estimated above, as shown in Fig.5A. The $\mathrm{PO}_{2}$ was initially decreasing linearly and then the decreasing rate slowed down. The first three data points were fitted by a linear relationship, as shown by the solid line, yielding the initial $\mathrm{PO}_{2} 149 \pm 8$ Torr and the oxygen consumption rate $0.14 \pm 0.01$ Torr s${ }^{-1}$. Since an extrapolation of the $\mathrm{PO}_{2}$ at the zero time gives about 150 Torr, the assumption of 120 Torr at the first data point is reasonable for this analysis. The oxygen concentration in a single cell is well traced during oxygen consumption by the GOx-Cat system.

The oxygen consumption by the respiration of cell itself was also measured on a confluent cultured dish. The consumption rate was very low, and eventually the experiments were mostly conducted under a high oxygen concentration. On the other hand, the measurements under a high oxygen concentration caused cell damages, resulting in a difficulty in the single cell tracing. Thus, after the measurement in a cell at a time point, the other cell was measured at the next time point. The oxygen concentration was obtained by fitting correlation function using Eqs.(4) and (6-8) with the averaged values of the decay times $(0.52$ and $0.09 \mathrm{~ms})$, the quenching constants $(0.11$ and $\left.0.48 \mathrm{~ms}^{-1} \mathrm{Torr}^{-1}\right)$ and the ratio of the pre-exponential factor of each component $\left(a_{1}^{0} / a_{0}^{0}=\right.$ 0.182). The estimated value of the absolute oxygen concentration at the initial time was larger than 150 Torr because of the large error of the quenching constants, which were largely dependent on the estimation of the decay time under aerobic condition. Thus, we discuss the relative change of the oxygen concentration, assuming that the average value of the $\mathrm{PO}_{2}$ at the initial time was 150 Torr, as shown in Fig.5B. The error bars are estimated from the error matrix of the leastsquares fitting. Under endogenous respiration, the oxygen concentration gradually decreased until a plateau level, where it is at a relatively high $\mathrm{PO}_{2}$ value. This level might be determined by the 
balance between an oxygen leak due to the imperfect sealing of chamber and the respiration rate. The initial oxygen consumption rate, $0.016 \pm 0.005 \% \mathrm{~s}^{-1}\left(0.024 \mathrm{Torr} \mathrm{s}^{-1}\right)$, was estimated by a linear fitting to the first four data points.

On the other hand, after an addition of a $1.4 \mu \mathrm{M} \mathrm{CCCP}$, an uncoupler of oxidative phosphorylation in the cell, the oxygen concentration rapidly decreased and then reached a slightly smaller oxygen concentration level than the level under the endogenous respiration as shown by the broken line in Fig.5B. The oxygen consumption rate was increased by CCCP.

\section{Discussion}

The absolute oxygen consumption was estimated from the cell density about $2.2 \times 10^{3}$ cells $\mathrm{mm}^{-2}$, the volume $140 \mu \mathrm{l}$ and surface area about $1.1 \times 10^{2} \mathrm{~mm}^{2}$ of the chamber. The rates without the uncoupler was 1.4 nmoles $\min ^{-1} / 10^{6}$ cells. The order of the oxygen consumption rate was near but small relative to the values observed in cells of other type at $37^{\circ} \mathrm{C}$.(Villani and Attardi, 1997) Although the quality of data is needed to be improved for the detail quantitative discussion, the oxygen concentration inside the cell can be measured by means of the correlation method.

Since HeLa cells could be easily stained by Pd:TSPP solution, other type of cells in tissue also may be stained if the Pd:TSPP solution can be applied to organs. Recently, it has been reported that the endogenous PpIX selectively accumulates in mitochondria and its delayed fluorescence can be used as an oxygen probe.(Mik et al., 2006) Thus, PpIX as an oxygen probe is also promising and our method is applicable to the PpIX measurements. Consequently, the oxygen concentration change inside the cell at different locations in the organ is possibly measured by this method. Furthermore, FCS measurements with the same apparatus may allow us to discuss interactions of 
oxygen concentration dependent factors when the temporal resolution is improved.

On the other hand, the current system could measure fluorescence correlation functions of fluorescent latex beads in a range of milliseconds and possibly can be used in blood flow measurements in vivo. Thus, both the flow and oxygen concentration measurements in vivo with this single setup can analyze the oxygen dynamics. In particular, the measurements in tumor under photodynamic diagnosis and therapy is one of the very interesting applications.(Glanzmann et al., 1999; Cubeddu et al., 2002) The simplicity is a great advantage when the use of the system is extended to clinical situations.

The correlation method presented here has an advantage in the measurement. Since the origin of the time means essentially nothing in the correlation measurements, the reference timing signal is not required and eventually this simplifies the system. The system using a microscope with a high numerical aperture objective lens can measure both the decay time in a cell and also the fluorescence correlation function. Although the higher average excitation pulse frequency is required in the efficient fluorescence correlation measurements, the measurements can be done with the single setup, including the excitation source. The use of a semi-conductor laser diode would contribute to the improvement of the method because the average excitation frequency can be easily changed. A cross-correlation detection of the signal also greatly improves the dead time and afterpulsing distortion of the correlation measurements, resulting a better time resolution down to in a 10 nano-seconds range.(Chirico and Gardella, 1999) This improvement will enable the decay measurements by the correlation method to be applicable to a more wider range.

This simple measurement is restricted in the multichromophore system and an extension of the theory connected to a single molecule level to the multichromophore system is required because 
the quantum nature of fluorescence has an important role in the single molecule level, resulting in the anti-bunching photons. Furthermore, the choice of the excitation frequency is also important. For the decay measurements, a higher frequency improves the statistics of data although the correlation amplitude becomes small. On the other hand, higher excitation frequency is required for the FCS measurements. The fluorescence molecule can be excited once within each excitation pulse when the duration of the excitation pulse is smaller than the order of fluorescence decay time. Consequently, when the interval between adjacent excitations becomes longer than the diffusion time, the correlation of the emission becomes very weak. These are also very important subjects to discuss the applicability of our method.

This method does not require the reference signal. This is an advantage in the practical medical application because it is simple. A fluorescence correlation measurement has been demonstrated, but the correlation method is not restricted to the fluorescence correlation. For example, the intensity correlation function of scattering light carries informations on the dynamics, absorption and scattering of tissue like medium.(Katayama et al., 1995; Nishimura et al., 1996; Durduran et al., 2005; Li et al., 2006) This simple method can combine such correlation measurements. The correlation method in luminescence decay measurement is expected to expand an applicability of the intensity correlation method. 


\section{References}

Bacia, K., Kim, S. A., Schwille, P., 2006. Fluorescence cross-correlation spectroscopy in living cells. Nat.Methods 3, 83-89.

Banks, D. D., Kerwin, B. A., 2004. Deoxygenation system for measuring protein phosphorescence. Anal.Biochem. 324, 106-114.

Bódis, E., Strambini, G. B., Gonnelli, M., Málnási-Csizmadia, A., Béla Somogyi, 2004. Characterization of f-actin tryptophan phosphorescence in the presence and absence of tryptophan-free myosin motor domain. Biophys.J 87, 1146-1154.

Chirico, G., Gardella, M., 1999. Photon cross-correlation spectroscopy to 10 ns resolution. Appl.Opt. 38, 2059-2067.

Chou, P.-T., Chi, Y., 2007. Phosphorescent dyes for organic light-emitting diodes. Chem. Eur. J. $13,380-395$.

Cubeddu, R., Comelli, D., D’Andrea, C., Toroni, P., Valentini, G., 2002. Time-resolved fluorescence imaging in biology and medicine. J.Phys.D 35, R61-R76.

Durduran, T., Choe, R., Yu, G., Zhou, C., Tchou, J. C., Czerniecki, B. J., Yodh, A. G., 2005. Diffuse optical measurement of blood flow in breast tumors. Opt.Lett. 30, 2915-2917.

Felekyan, S., Kühnemuth, R., Kudryavtsev, V., Sandhagen, C., Becker, W., Seidel, C. M., 2005. Full correlation from picoseconds to seconds by time-resolved and time-correlated single photon detection. Rev. Sci. Instrum. 76, 083104. 
Földes-Papp, Z., 2006. What it means to measure a single molecule in a solution by fluorescence fluctuation spectroscopy. Exp.Mol.Path. 80, 209-218.

Glanzmann, T., Ballini, J.-P., van den Bergh, H., Wagniéres, G., 1999. Time-resolved spectrofluorometer for clinical tissue characterization during endoscopy. Rev.Sci.Instrum. 70, 4067-4077.

Katayama, K., Nishimura, G., Kinjo, M., Tamura, M., 1995. Absorbance measurements in turbid media by the photon correlation method. Appl.Opt. 34, 7419-7427.

Lakowicz, J. R., 1999. Principles of Fluorescence Spectroscopy. Kluwer Academic/Plenum Press, New York and London.

Li, J., Jaillon, F., Dietsche, G., Maret, G., Gisler, T., 2006. Pulsation-resolved deep tissue dynamics measured with diffusing-wave spectroscopy. Opt.Exp. 14, 7841-7851.

Mik, E. G., Stap, J., Sinaasappel, M., Beek, J. F., Aten, J. A., van Leeuwen, T. G., Ince, C., 2006. Mitochondrial $\mathrm{PO}_{2}$ measured by delayed fluorescence of endogenous protoporphyrin IX. Nature Methods 3, 939-945.

Mik, E. G., van Leeuwen, T. G., Raat, N. J., Ince, C., 2004. Quantitative determination of localized tissue oxygen concentration in vivo by two-photon excitation phosphorescence lifetime measurements. J.Appl.Physiol. 97, 1962-1969.

Nishimura, G., Katayama, K., Kinjo, M., Tamura, M., 1996. Diffusing-wave absorption spectroscopy in the homogeneous turbid media. Opt.Communi 126, 99-107.

Nishimura, G., Pack, C., Tamura, M., 2006. Expansion of intensity correlation spectroscopy 
for lifetime measurements - application to intracellular oxygen dynamics measurements. J.Biomed.Opt. (in press).

Pravinata, L. C., You, Y., Ludescher, R. D., 2005. Erythrosin b phosphorescence monitors molecular mobility and dynamic site heterogeneity in amorphous sucrose. Biophys.J 88, 3551-3561.

Rigler, R., Elson, E. S., 2001. Fluorescence Correlation Spectroscopy. Theory and Applications. Springer-Verlag, Berlin.

Vanderkooi, J. M., Maniara, G., Green, T. J., Wilson, D. F., 1987. An optical method for measurement of dioxygen concentration based upon quenching of phosphorescence. J.Biol.Chem. 262, $5476-5482$.

Villani, G., Attardi, G., 1997. In vivo control of respiration by cytochrome c oxidase in wild-type and mitochondrial dna mutation-carrying human cells. Proc.Nat.Acad.Sci. USA 94, 1166-1171.

Vukojevi, V., Pramanik, A., Yakovleva, T., Rigler, R., Terenius, L., Bakalkn, G., 2005. Study of molecular events in cells by fluorescence correlation spectroscopy. Cell. Mol. Life Sci. 62, $535-550$.

Wilson, D. F., Vinogradov, S. A., 2003. Tissue oxygen measurements using phosphorescence quenching. In: Mycek, M.-A., Pogue, B. W. (Eds.), Handbook of Biomedical Fluorescence. Marcel Dekker, Inc., New York, Ch. 17, pp. 637-662. 


\section{Appendix}

The correlation function of the emission is given as follows. The intensity of the emission was expressed by $I(t)=I_{0} \sum_{i} F\left(t-t_{i}\right)$ when the $i$-th excitation light is coming at time $t_{i}$, where $I_{0}$ and $F(t)$ are the appropriate normalization constant and the decay function of system, respectively. Using $F(t)$, the correlation function $\langle I(t) I(t+\tau)\rangle$ is expressed as

$$
\langle I(t) I(t+\tau)\rangle=I_{0}^{2}\left\langle\sum_{i, j} F\left(t-t_{i}\right) F\left(t+\tau-t_{j}\right)\right\rangle .
$$

If the timing of the excitation is randomized, the emission only correlates within the emissions excited by the same single excitation pulse, and thus it can be simplified as

$$
\langle I(t) I(t+\tau)\rangle=I_{0}^{2}\left\langle\sum_{i} F\left(t-t_{i}\right) F\left(t+\tau-t_{i}\right)\right\rangle+I_{0}^{2}\left\langle\sum_{i} F\left(t-t_{i}\right)\right\rangle^{2}
$$

The ensemble average of a function $A(t)$ can be replaced by a time average as

$$
\langle A(t)\rangle=\lim _{T \rightarrow \infty} \frac{1}{T} \int_{-T / 2}^{T / 2} A(t) d t .
$$

When $A(t)=\sum a\left(t-t_{i}\right)$ where $a(t)$ rapidly decays, the ensemble average is given by

$$
\langle A(t)\rangle=v \lim _{T \rightarrow \infty} \int_{-T / 2}^{T / 2} a(t) d t
$$

where $v$ is the average excitation rate given by $\left\langle\left(t_{i}-t_{i-1}\right)\right\rangle=1 / v$. Eventually, the correlation function can be expressed as

$$
\frac{\langle I(t) I(t+\tau)\rangle}{\langle I(t)\rangle^{2}}=\left[v I_{0}^{2} \int_{0}^{\infty} F(t) F(t+\tau) d t\right] /\left[v^{2} I_{0}^{2}\left(\int_{0}^{\infty} F(t) d t\right)^{2}\right]+1,
$$

Assuming a multiexponential decay kinetics $F(t)=\sum_{i} a_{i} / \tau_{i} \exp \left(-t / \tau_{i}\right)$ where $\sum_{i} a_{i}=1$, the correlation function

$$
g(\tau)=\frac{1}{v} \sum_{i}\left(\sum_{j} \frac{a_{j}}{1+\tau_{j} / \tau_{i}}\right) \frac{a_{i}}{\tau_{i}} \exp \left(-\frac{\tau}{\tau_{i}}\right)
$$


can be yielded.

The decay time of each decay component in the correlation function is identical to the decay time in the decay function of emission. On the other hand, the pre-exponential factor is not identical to the pre-exponential factor in the decay function and dependent on both the pre-exponential factors $a_{i}$ and the decay times $\tau_{i}$. 


\section{Figure Legends}

Fig.1 Decay measurements in vitro. Phosphorescence decay curves (A) in Pd:TSPP-BSA conjugate solution and (B) in Pd:TSPP solution with the GOx-Cat system in a sealed sample chamber. The correlation functions from top to bottom are the results with decreasing in oxygen concentration. (C) Correlation amplitude as a function of the decay time. Open and filled circles denote the results with Pd:TSPP and Pd:TSPP-BSA conjugate solutions, respectively.

Fig.2 The fluorescence correlation measurements with fluorescent beads in PBS. (A) Typical fluorescence correlation function (circles) and the fitting results with two-component model (line). Inset shows the same correlation function with wider time scale. (B) The number of particles against the concentration of fluorescent beads.

Fig.3 Measurements with HeLa cells stained by Pd:TSPP. (A) LSM image of phosphorescence under aerobic condition. (B) A far field transmission image with a laser excitation spot. The arrow indicates an excitation point in cytoplasm. (C) Typical decay curves of the phosphorescence correlation function. The circles and boxes show the decay curves under aerobic and anaerobic conditions, respectively. The oxygen in the medium in the incubation chamber sealed by a cover glass was consumed by the GOx-Cat system to maintain the anaerobic condition. The solid lines denote the fitting results by a double exponential function.

Fig.4 The double exponential analysis of the phosphorescence decay in HeLa cell under aerobic $(A, B)$ and anaerobic $(C, D)$ conditions. Each symbol shows result in a different cell and the error bars show the standard deviation estimated by the error matrix of the least-squares 
fitting. The open circles and filled boxes denote the slow and fast components of the fitting, respectively.

Fig.5 The oxygen concentration in HeLa cells. (A) The oxygen consumed by using the GOx-Cat system. Inset shows raw correlation curves at 175, 842, 1092, 1292, 1492, and $2318 \mathrm{~s}$ after GOx addition. (B) The oxygen consumed by respiration of HeLa cells without and with an uncoupler CCCP. The error bars show the standard deviation estimated from the error matrix of the least-squares fitting. 

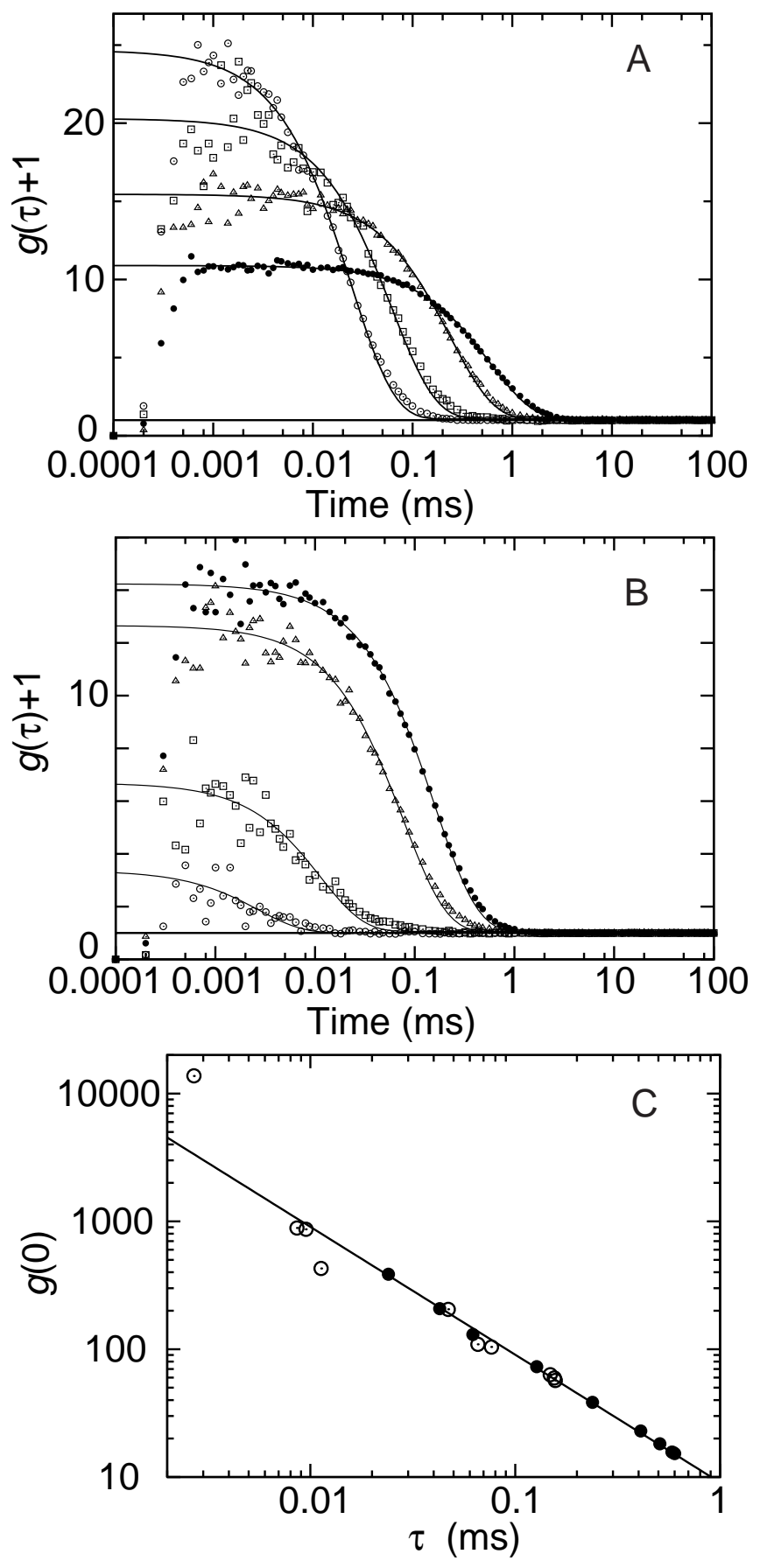

Figure 1: 

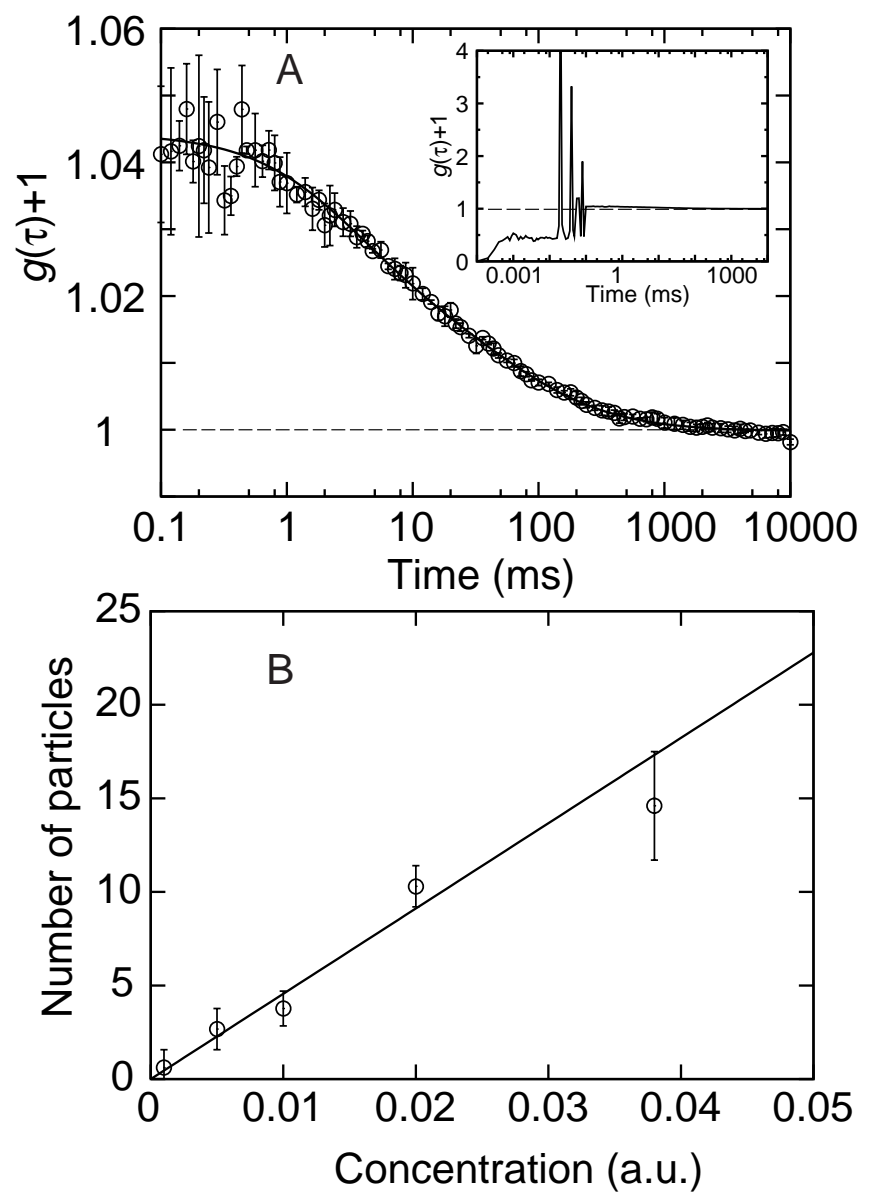

Figure 2: 

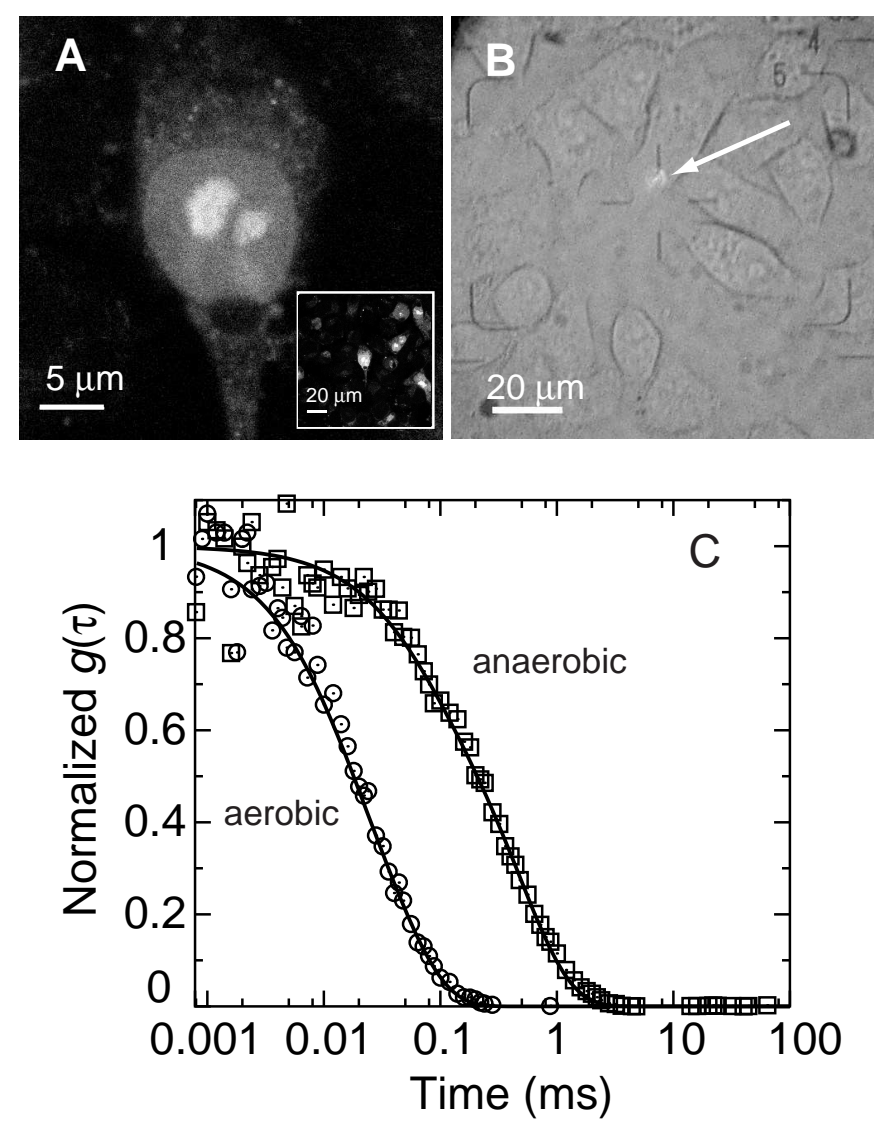

Figure 3: 

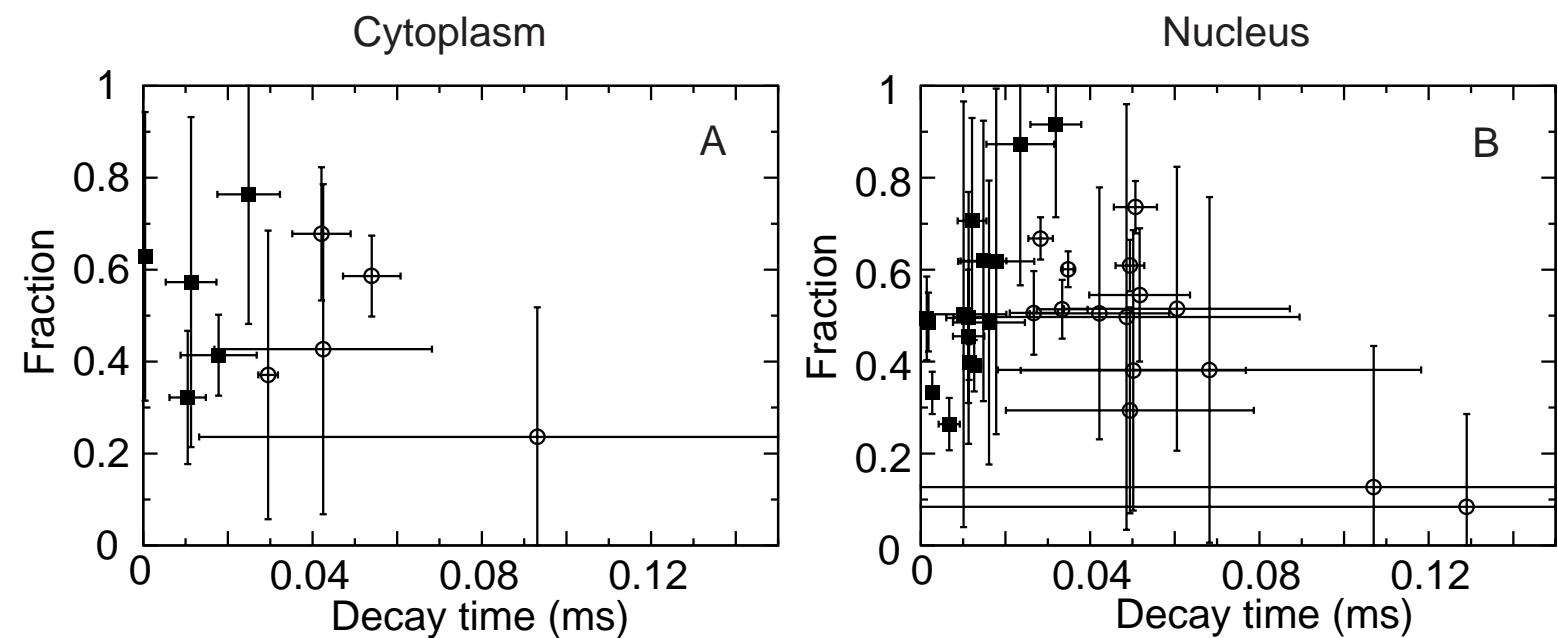

aerobic
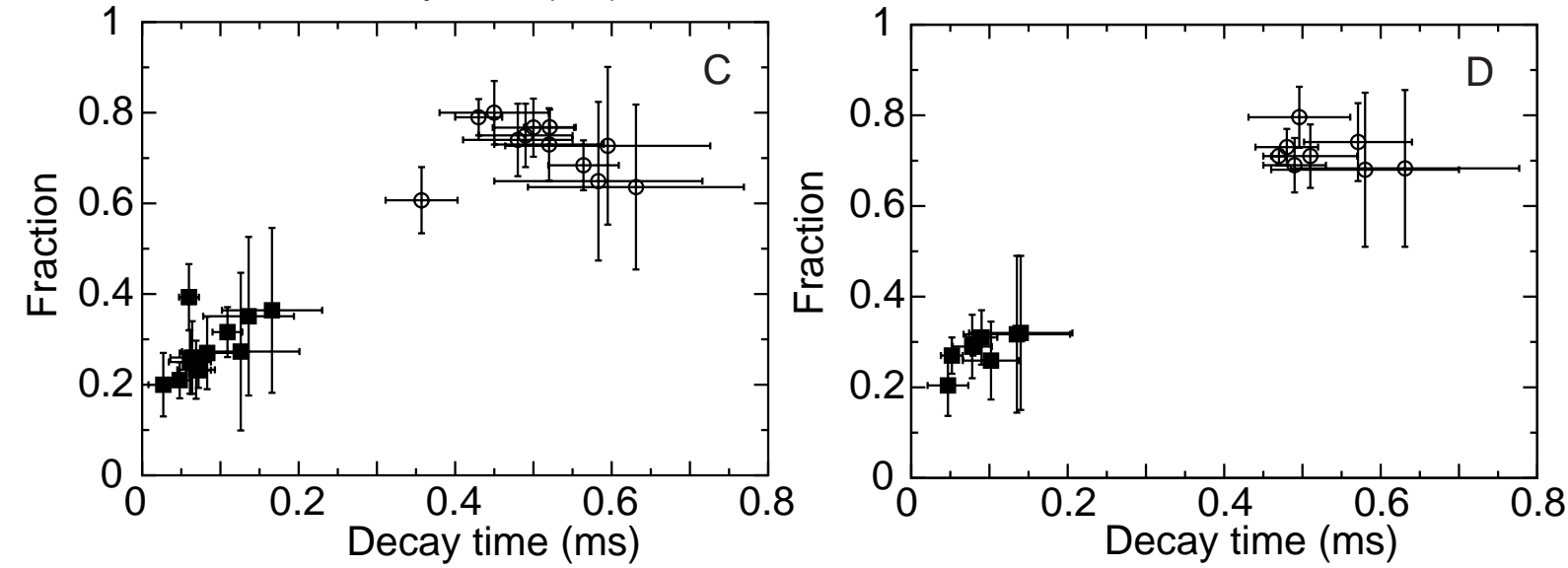

anaerobic 


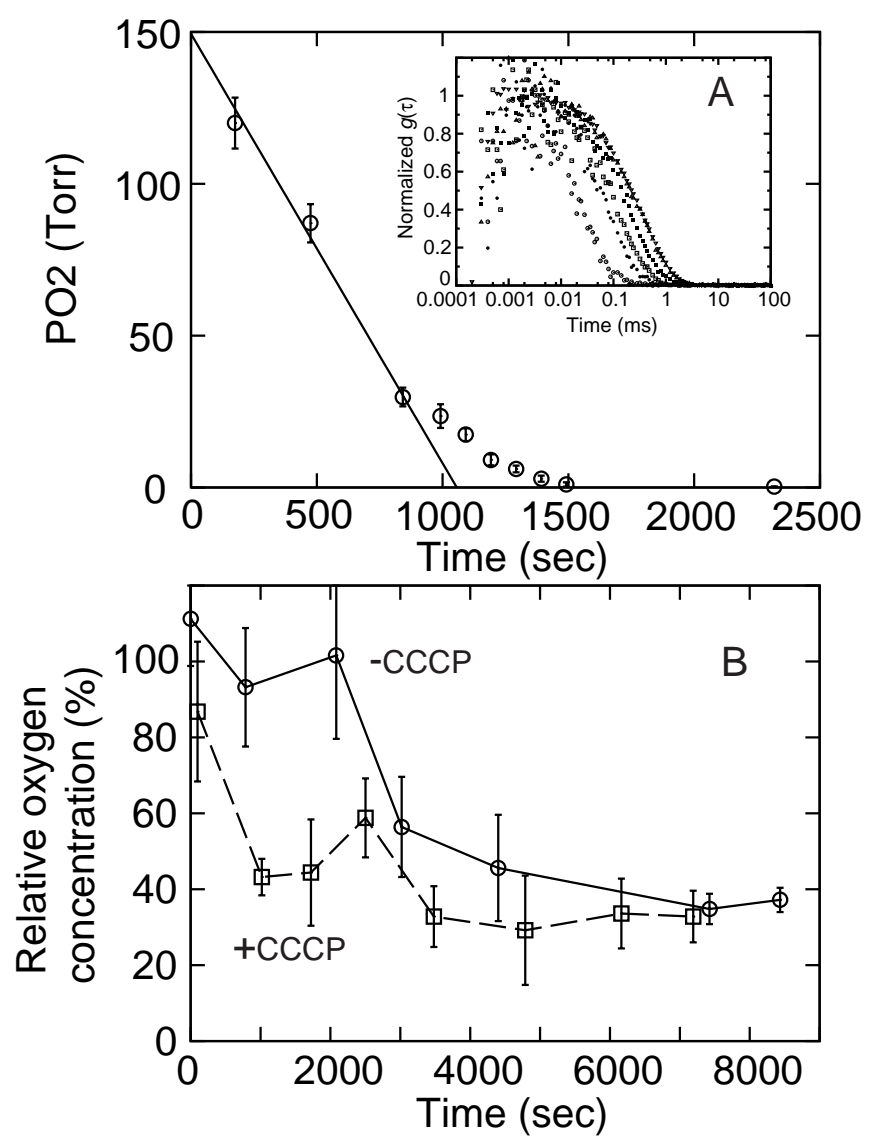

Figure 5: 